\title{
Development of Argatroban as an Anticoagulant and Antithrombin Agent in Japan
}

\author{
Hidenobu Ikoma \\ Development Coordination Department, Pharmaceuticals Development Unit, Research and Development Division, \\ Mitsubishi Pharma Corporation, Tokyo, Japan
}

\section{Key Words}

Argatroban - Direct thrombin inhibitor .

Cerebral thrombosis - Chronic arterial occlusion . Hemodialysis in AT deficiency

\begin{abstract}
Argatroban is a synthetic direct thrombin inhibitor which has been used in Japan for three indications, namely, chronic arterial occlusion, acute cerebral thrombosis and hemodialysis in AT-deficient patients or in patients with decreased AT. In patients with chronic arterial occlusion, argatroban increased the skin temperature, reduced the size of skin ulcers, and decreased the thrombin-antithrombin complex. In patients with acute cerebral thrombosis, neuronal symptoms improved significantly compared with the placebo group, wherein activated partial thromboplastin time (aPTT) was prolonged by about 1.5fold and fibrinopeptide $A$ was reduced. In some patients in whom hemodialysis is difficult due to the generation of blood clots in the dialyzer, hemodialysis is now possible with the replacement of heparin by argatroban.
\end{abstract}

Copyright $@ 2002$ S. Karger AG, Basel

\section{Introduction}

Argatroban has been approved for three indications in Japan. In 1990, argatroban was approved as an anticoagulant for chronic arterial occlusion. In 1996, approval was granted for the additional indications of acute cerebral thrombosis and for the treatment of hemodialysis patients who are antithrombin (AT) deficient or with decreased AT.

Outside Japan, argatroban was approved by the FDA as an anticoagulant for the prophylaxis or treatment of thrombosis in patients with heparin-induced thrombocytopenia (HIT). Argatroban has been launched in Korea for chronic arterial occlusion and acute cerebral thrombosis. Argatroban has also been approved for use in Canada.

This paper provides an overview of the history of the development of argatroban in Japan and the effect of the drug on coagulation tests in the three indications of acute cerebral thrombosis, chronic arterial occlusion, and hemodialysis in AT-deficient patients or patients with decreased AT. Details of the approved indications in Japan are as follows:

(1) acute cerebral thrombosis: improvement of neurological symptoms (paralysis) accompanying the disease and improvement of activities of daily living (ADL)

\begin{tabular}{ll}
\hline KARGER & ( ) 2002 S. Karger AG, Basel \\
Fax +4161306 1234-8832/02/0329-0023\$18.50/0 \\
$\begin{array}{l}\text { E-Mail karger@karger.ch } \\
\text { www.karger.com }\end{array}$ & $\begin{array}{l}\text { Accessible online at: } \\
\text { www.karger.com/pht }\end{array}$
\end{tabular}

Hidenobu Ikoma, Clinical Development Department 1

Research and Development Division, Mitsubishi-Tokyo Pharmaceutical, Inc.

2-2-6 Nihonbashi-Honcho, Chuo-ku, Tokyo 103-8405 (Japan)

Tel. +8133242 4391, Fax +81332414807

E-Mail Ikoma.Hidenobu@mh.m-pharma.co.jp 
(which involves the ability to walk, stand up, maintain a sitting position, and take meals); (a) acute cerebral thrombosis (excluding lacunes) within $48 \mathrm{~h}$ after onset;

(2) improvement in limb ulcers, rest pain, and a sensation of cold accompanying chronic arterial occlusion (Buerger's disease, arteriosclerosis obliterans);

(3) prevention of clotting in the perfused blood during extracorporeal circulation in the following patients: (a) congenital AT-deficient patients or patients with decreased AT; (b) patients with reduced AT levels (patients where AT is $<70 \%$ normal values or where heparin treatment produces no improvement in clotting (residual blood) during extracorporeal circulation).

\section{History of Development in Japan}

Thrombosis is thought to occur as a result of an imbalance in the blood coagulation-fibrinolytic system that produces a tendency to clot. Thrombin plays a very important role in the blood coagulation system. Thrombin not only converts fibrinogen to fibrin, but it also initiates platelet aggregation, and blood vessel constriction.

Research was started in this area on the basis that substances that suppress the thrombus-forming activity of thrombin may be promising in drug therapy for thrombosis. In 1972, research by Mitsubishi Chemical Industries and Okamoto et al. [1] showed that thrombin promotes the conversion of fibrinogen to fibrin by cutting the ArgGly bond in fibrinogen. Starting with a thrombin inhibitor, tosyl arginyl methyl ester, research began on the development of a potent and selective antithrombin drug through the substitution of the arginine carbonyl group and amino group.

The results of this research showed that argatroban inhibited thrombin at extremely low concentrations and that the drug had an extremely weak inhibitory effect on trypsin (also a serine protease), factor Xa, plasmin, and kallikrein [2]. These results confirmed the concept that argatroban selectively inhibits thrombin.

\section{Pharmacological Effects of Argatroban}

Argatroban binds reversibly to thrombin and its effects are short-lived [3]. The therapeutic effects of argatroban can be easily monitored by anticoagulation tests (such as aPTT or prothrombin time (PT)), so anticoagulation therapy with argatroban is easy to control. Argatroban is highly selective for thrombin. The molecular weight is low
(527 Da). Thus, argatroban can bind not only to circulating thrombin but also to clot-bound thrombin, unlike heparins that bind solely to circulating thrombin [4]. Argatroban does not require the cofactor AT for antithrombotic activity, in contrast to heparins and heparinoids [5]. Thus, argatroban can exert its effect even in AT-deficient patients and in patients with decreased AT [6]. Finally, unlike heparins, argatroban does not generate antibodies and, unlike heparinoids and low molecular weight heparins, argatroban does not cross-react with heparin antibodies [7].

In addition to the pharmacological profile shown above, argatroban demonstrates several other important actions. In vitro studies have shown that argatroban inhibits thrombin-mediated endothelin-1 release from the endothelium [8], inhibits thrombin-mediated vascular constriction [9], and increases the effect of thrombolytic agents such as tissue plasminogen activator (t-PA) [10]. Moreover, in terms of its anticoagulant action, argatroban differs from heparin in vitro in that it produces no excessive prolongation of the aPTT in a wide range of concentrations, while heparin produces marked prolongation of aPTT at a narrow range of concentrations [11] (fig. 1). Intravenous administration of heparin (1,000 IU) or argatroban $(6 \mathrm{mg})$ for $2 \mathrm{~h}$ produces an exponential prolongation of aPTT during the infusion with heparin, but a plateau at approximately $30 \mathrm{~min}$ after commencing the argatroban infusion [12] (fig. 1). Thus, there is considerable control of coagulation during the argatroban infusion.

\section{Argatroban and Chronic Arterial Occlusion}

In chronic arterial occlusion, for which argatroban is indicated, the thrombus forms in the blood vessel lumen and repeatedly exacerbates or rekindles ischemic symptoms. In addition to ischemic symptoms such as cold, cyanosis, rest pain, and intermittent claudication, the disease can worsen limb ulcers and many cases require amputation.

A pivotal study [13] using a double dummy method compared with argatroban (twice daily doses of $10 \mathrm{mg}$ given by intravenous infusion over $3 \mathrm{~h}$, for 4 weeks) with the antiplatelet agent ticlopidine $(500 \mathrm{mg} /$ day by oral administration for 6 weeks). Results in the argatroban group were equivalent to those in the panaldine group, in terms of degree of ulcer reduction and improvement in rest pain and cold. Argatroban, however, has a rapid onset of action and is effective in cases not improved by treatment with
Ikoma 

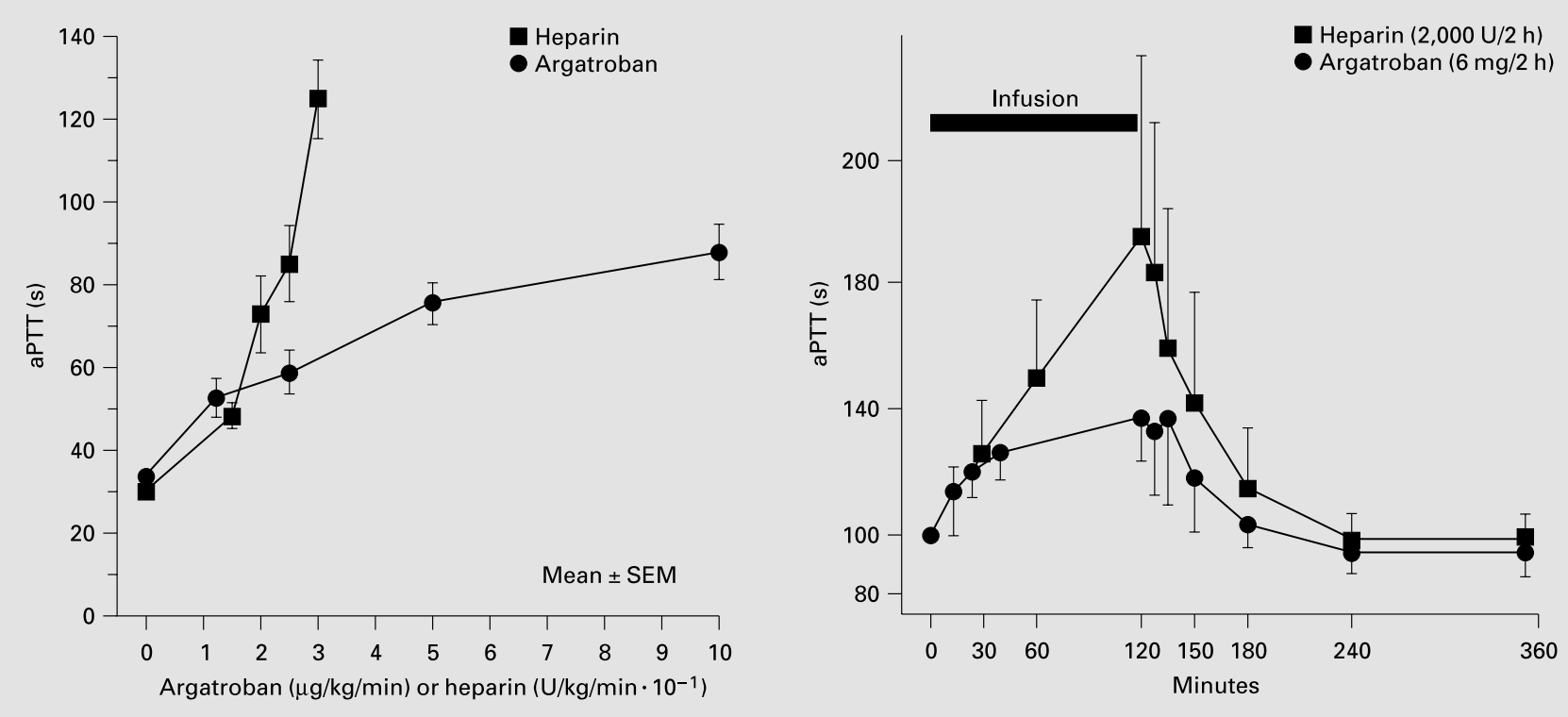

Fig. 1. Effects of continuous infusions $(2 \mathrm{~h})$ of heparin or argatroban on aPTT in healthy subjects. From Fukutake et al. [12] (with slight modification).

Fig. 2. Transcutaneous tissue oxygen partial pressure $\left(\mathrm{TcPO}_{2}\right)$ of skin surface of a patient with chronic arterial occlusion. From Itoh et al. [14] (with slight modification).

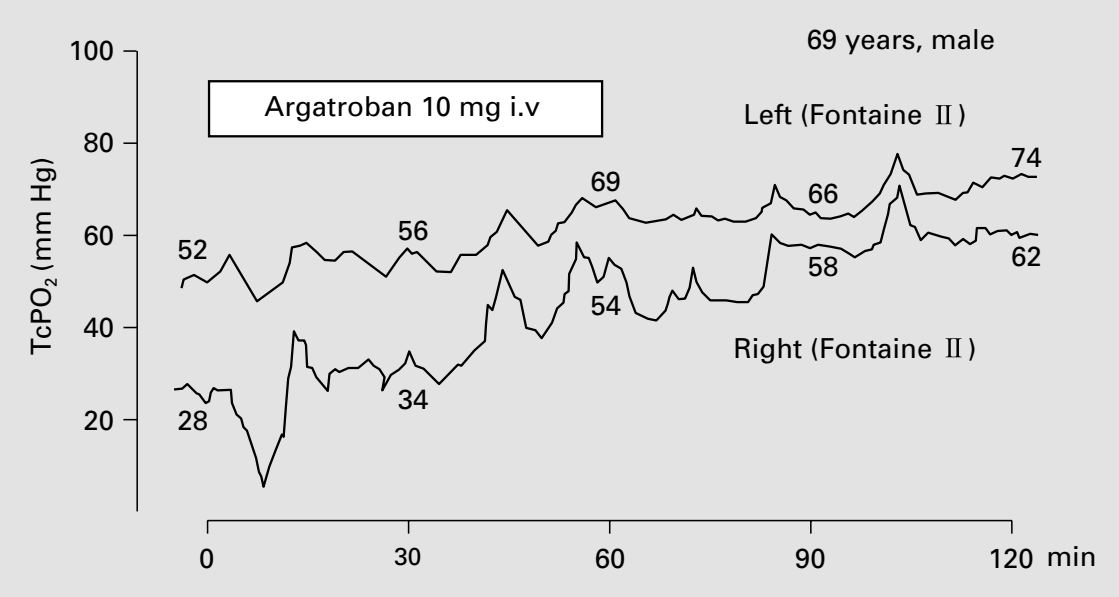

antiplatelet, vasodilators, or other agents. Moreover, when $10 \mathrm{mg}$ argatroban was administered intravenously for $1 \mathrm{~h}$, the drug increased the transcutaneous tissue oxygen partial pressure $\left(\mathrm{TcPO}_{2}\right)$ at the skin surface of the diseased leg more than the healthy leg (fig. 2). The results also confirmed increased skin temperature and $\mathrm{TcPO}_{2}$ in the diseased leg in severe cases, compared with mild cases [14]. This effect persisted regardless of the low blood con- centration of argatroban after infusion. However, heparin was shown to have almost no influence on the peripheral circulation.

In patients with chronic arterial occlusion, argatroban produced a decrease in thrombin-antithrombin complex (TAT), which is an indicator of thrombin formation [15]. TAT levels after argatroban therapy were decreased in patients with chronic arterial occlusion. The decrease 

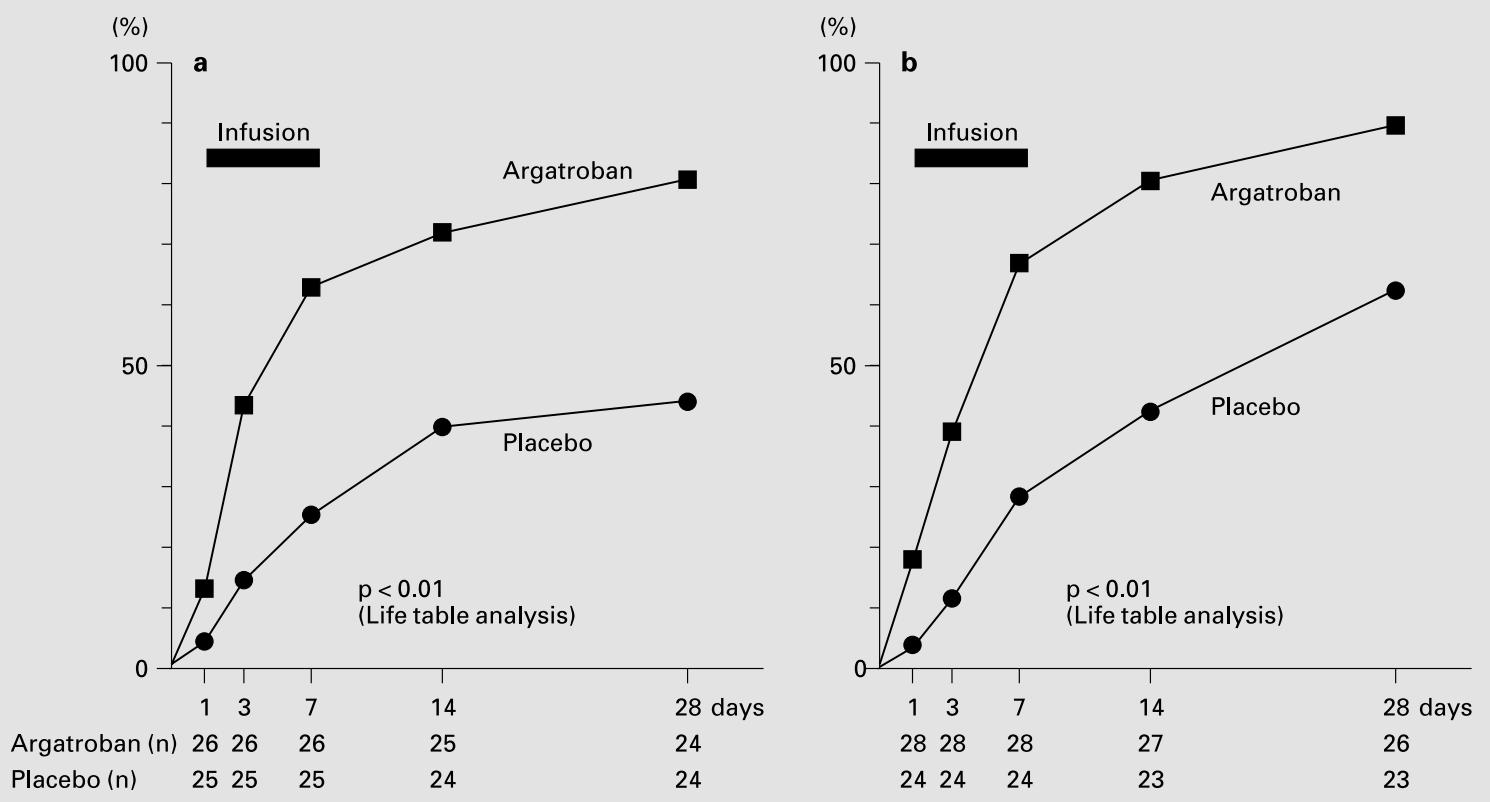

Fig. 3. Percentage of patients who showed more than one rank improvement in the symptom scale during treatment a Paralyzed leg scale. The degree of muscle power on the motor paralyzed side was classified into the following 4 grades: 0 : normal; 1 : can move ankle against gravity; 2 : extended leg raising or knee flexion possible; 3 : can move only on floor, or unable to move at all. b ADL scale. ADL was classified into the following 4 grades: 0: normal (no symptoms); 1 : mild (can care almost by oneself, but slow in action); 2: moderate (needs assistance); 3: severe (cannot perform at all). From Kobayashi et al. [18] (with slight modification). in plasma TAT levels after argatroban therapy indicates an improvement in the hypercoagulable state.

\section{Argatroban and Acute Cerebral Thrombosis}

In acute cerebral thrombosis, another argatroban indication, the thrombus forms at the site of a vascular lesion producing occlusive symptoms, and new symptoms occur over time after the initial onset of symptoms, accompanying enlargement of the ischemic area. Symptoms usually stabilize after several hours or days [16].

This concept is supported by reports of the following research into coagulation after symptom onset. A study in patients after the onset of cerebral thrombosis has shown that fibrinopeptide A (FPA), an indicator of thrombin activity, increases until 7 days after symptom onset, with a peak at 3 days after onset [17]. In cerebral infarction patients and cerebral thrombosis models after thrombotic occlusion, there is secondary formation of minute thrombi over time, mainly involving fibrin [16]. This impairs the microcirculation, resulting in continued thrombin production in the acute phase after symptom onset and may be involved in the hypercoagulable state.

Symptomatic improvement may be due to argatroban preventing the progression of the infarct into the ischemic penumbra.

In acute cerebral thrombosis, a pivotal study on argatroban compared the drug with placebo in a double-blind trial [18]. Patients were given continuous drip infusions for the first 2 days $(60 \mathrm{mg} /$ day $)$ followed by twice-daily infusions $(10 \mathrm{mg} \times 2)$ for $3 \mathrm{~h}$ each on days 3-7. In comparison with placebo, a significant difference was seen in the argatroban group in terms of improvement of neurological symptoms (upper and lower paralysis-paralysed leg scale) and ADL (ability to walk, stand up, maintain a sitting position, eat) (fig. 3). The study showed that aPTT was prolonged by approximately 1.5 times and that FPA 

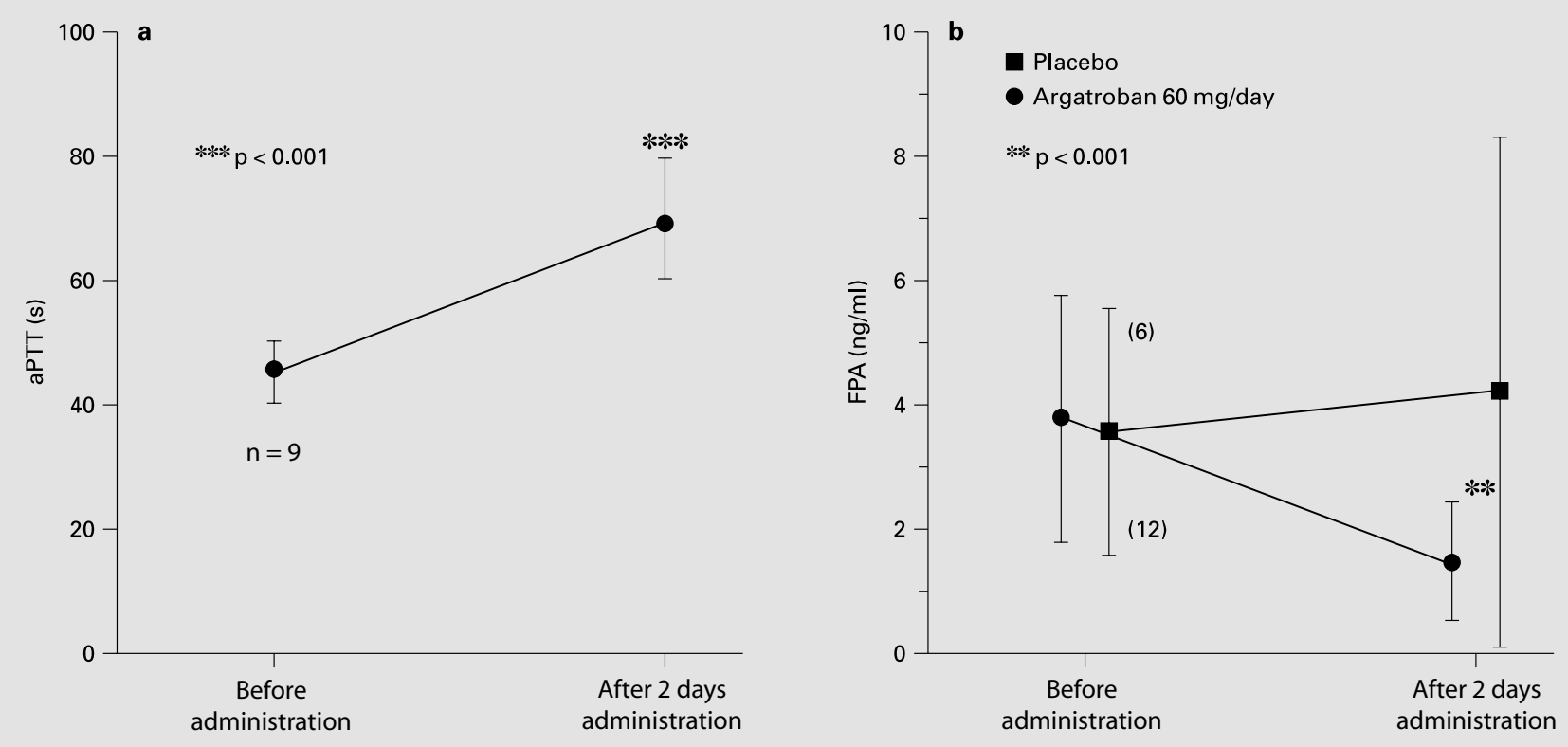

Fig. 4. Effect of a 120-mg continuous drip infusion of argatroban for $48 \mathrm{~h}$ on aPTT (a) and FPA (b) (means \pm SD) in acute cerebral thrombosis. The number of cases is shown in parentheses. From Tanaka et al. [19] (with slight modification).

levels, which were high before argatroban administration, were reduced to normal levels after drug administration [19] (fig. 4). The normalization of FPA levels following argatroban administration indicates that argatroban inhibited thrombin in the blood and improved the hypercoagulable state immediately after the onset of cerebral thrombosis. This activity may be linked to the subsequent symptomatic improvement.

\section{Argatroban and Hemodialysis in AT-Deficient Patients or Patients with Decreased AT}

Heparin is commonly used as an anticoagulant in hemodialysis. Heparin exerts its anticoagulant activity by promoting binding of thrombin and AT in the blood, so the antithrombotic activity of heparin is reduced in patients with $<70 \%$ normal levels of blood AT activity. There have been reports that heparin does not produce the expected anticoagulant activity in AT-deficient patients or patients with decreased AT, especially in hemodialysis where clotting in the dialyzer makes hemodialysis extremely difficult to maintain [6]. In contrast to heparin, the anticoagulant action of argatroban does not involve AT and the drug can exhibit antithrombin activity and the resulting anticoagulant activity without exhausting AT. Argatroban was considered to have potential in patients with congenital or acquired AT deficiency, where hemodialysis proves extremely difficult with heparin treatment.

In patients where hemodialysis is extremely difficult with heparin treatment because of coagulation in the dialyzer, argatroban administered within the dialysis circuit at $12-48 \mathrm{mg} / \mathrm{h}$ through continuous drip infusion was confirmed as effective in preventing clotting in the dialyzer [6].

\section{Argatroban and Post-Marketing Surveillance Safety Information in Japan}

Table 1 shows the summarized results of post-marketing surveillance safety information of argatroban in Japan. During a five-year period there were approximately 3,800 cases on each indication of acute cerebral thrombosis or chronic arterial occlusion treated with argatroban. 
Table 1. Post-marketing surveillance of argatroban in Japan

\begin{tabular}{lcc}
\hline Survey items & Stroke & $\begin{array}{l}\text { Chronic } \\
\text { arterial } \\
\text { occlusion }\end{array}$ \\
\hline Institutions included & 590 & 620 \\
Cases examined & 3,777 & 3,791 \\
Cases with adverse reactions & $386(10.2 \%)$ & $178(4.7 \%)$ \\
Hemorrhagic adverse events & $77(2.0 \%)$ & $33(0.9 \%)$ \\
$\quad$ Hemorrhagic cerebral infarction & $44(1.2 \%)$ & 0 \\
$\quad$ Hematuria & $12(0.3 \%)$ & $10(0.3 \%)$ \\
$\quad$ Gastrointestinal hemorrhage & $5(0.1 \%)$ & $3(0.1 \%)$ \\
$\quad$ Cerebral hemorrhage & $3(0.1 \%)$ & $2(0.1 \%)$ \\
$\quad$ Others & $13(0.3 \%)$ & $18(0.5 \%)$ \\
Nonhemorrhagic adverse events & $309(8.2 \%)$ & $145(3.8 \%)$ \\
\hline
\end{tabular}

These cases were examined. The most significant adverse events for argatroban are hemorrhagic complications, but they were infrequent. Hemorrhagic cerebral infarction in stroke patients was observed in only 44 cases $(1.2 \%)$ and was not seen in patients with chronic arterial occlusion. Therefore, we have concluded that argatroban has an acceptable safety profile.

\section{Conclusion}

Overall, we think that the results obtained to date support the position that argatroban is an effective anticoagulant in a range of conditions and that the drug has an acceptable safety profile.

\section{References}

1 Okamoto S, Hijikata A, Kinjo K, Kikumoto R, Ohkubo K, Tonomura S, Tamao Y: A novel series of synthetic thrombin-inhibitors having extremely potent and highly selective action. Kobe J Med Sci 1975;21:43-51.

2 Okamoto S, Hijikata A, Kikumoto R, Tonomura S, Hara H, Ninomiya K, Maruyama A, Sugano M, Tamao Y: Potent inhibition of thrombin by the newly synthesized arginine derivative No. 805 The importance of stereostructure of its hydrophobic carboxamide portion. Biochem Biophys Res Commun 1981;101:440446.

3 Nagasawa H, Fukutake K, Hada M, Takahashi E, Matsubara Y, Samori T, Ikematsu S, Kitahara T, Ukita M, Fujimaki M, Fukutake K: Phase one study of synthetic antithrombin agent (MD-805). Single and multiple administration studies. Jpn J Clin Pharmacol 1981;12:359_ 375.

4 Berry CN, Girardot C, Lecoffre C, Lunven C: Effects of the synthetic thrombin inhibitor argatroban on fibrin- or clot-incorporated thrombin: Comparison with heparin and recombinant hirudin. Thromb Haemost 1994:72:381386.

5 Kumada T, Abiko Y: Comparative study on heparin and a synthetic thrombin inhibitor IIIdeficient animals. Thromb Res 1981;24:285298.
6 Ohta K, Koshikawa S, Hirasawa S, Mitsuno K, Hibi I, Sawada K, Sakai R, Matsuo T: Effectiveness of MD-805 (argatroban) in hemodialysis patients with antithrombin III deficiency. New Drugs Clin 1992;41:971-983.

7 Warkentin TE: Heparin-induced thrombocytopenia: A clinicopathologic syndrome. Thromb Haemost 1999;82:439-447.

8 Maruyama S: Synthetic anticoagulant. Jpn J Clin Hematol 1990;31:776-781.

9 Nakamura K, Hatano Y, Mori K: Thrombininduced vasocontriction in isolated cerebral arteries and the influence of a synthetic thrombin inhibitor. Thromb Res 1985;40:715-720.

10 Tamao Y, Yamamoto T, Kikumoto R, Hara H, Itoh J, Hirata T, Mineo K, Okamoto S: Effect of a selective thrombin inhibitor MCI-9038 on fibrinolysis in vitro and in vivo. Thromb Haemost 1986;56:28-34.

11 Tamao Y, Yamamoto T, Hirata T, Kinugasa M, Kikumoto R: Effect of argatroban on blood coagulation. Jap J Pharm Ther 1986;14:7-12.

12 Fukutake K, Tateyama M, Amano K, Ueda M, Fujimaki M: Clinical pharmacology of argatroban, selective anti-thrombin agent: Comparative study with heparin. Cardioangiology 1991; 29:190-196.

13 Tanabe T, Yasuda K, Mishima Y, Furukawa K, Sakaguchi S, Kamiya K, Shionoya S, Katsumura T, Kusaba A, Sakuma A: Therapeutic effect of argatroban (MD-805), a thrombin inhibitor, on chronic arterial occlusion. A comparative study with ticlopidine by a doubleblind study. Cardioangiology 1987;22:185202.
14 Itoh K, Ohgi S, Tanaka K, Tsujimoto M, Hara H, Mori T: The effect of MD 805, synthetic thrombin inhibitor, on peripheral circulation. Geka Shinryou (Tokyo) 1987;29:122-126.

15 Akahori M, Chishiro T, Tanaka T: Clinical discussion on the dose effect of argatroban in arteriosclerosis obliterans (ASO) - Especially coagulation and fibrinolysis marker Jpn J Med Pharm Sci 1993;30:409-405.

16 Sampaolo S, Cervos-Navarro J, Djouchadar D, Figols J: Clinical and experimental evidence of microthrombosis in cerebral ischemia; in Hartmann A, Wuschinesky (eds): Cerebral Ischemia and Hemorrheology. Berlin, Springer, 1987, pp 386-393.

17 Chao-Hung H, Han-Hwa H, Wen-Jang W: The serial hemostasis-related changes in patients with cerebral infarction: Comparison between progressing and non-progressing stroke. Thromb Res 1989;55:635-647.

18 Kobayashi S, Tazaki Y: Effect of the thrombin inhibitor Argatroban in acute cerebral thrombosis. Semin Thromb Hemost 1997;23:531534.

19 Tanaka Y, Kawabata S, Sin R, Takatsuka K, Nishikawa H, Yoshikawa N, Komatsu T: Therapeutic effect of argatroban (MD-805), antithrombin agent, in the acute stage of cerebral thrombosis. J Clin Ther Med 1987;3:133-142. 